\title{
Malignant solitary pulmonary nodules: assessment of mass growth rate and doubling time at follow-up CT
}

\author{
Jingxu Li*, Tingting Xia*, Xinguan Yang, Xiao Dong, Jiamin Liang, Nanshan Zhong, Yubao Guan
}

The First Affiliated Hospital of Guangzhou Medical University, State Key Laboratory of Respiratory Disease, National Clinical Research Center for Respiratory Disease, Guangzhou 510120, China

Contributions: (I) Conception and design: J Li, T Xia, Y Guan, N Zhong; (II) Administrative support: Y Guan, N Zhong; (III) Provision of study materials or patients: J Li, T Xia, X Yang; (IV) Collection and assembly of data: X Dong, J Liang, X Yang; (V) Data analysis and interpretation: T Xia, J Li, Y Guan, N Zhong; (VI) Manuscript writing: All authors; (VII) Final approval of manuscript: All authors.

*These authors contributed equally to this work.

Correspondence to: Yubao Guan; Nanshan Zhong. The First Affiliated Hospital of Guangzhou Medical University, State Key Laboratory of Respiratory Disease, National Clinical Research Center for Respiratory Disease, Guangzhou 510120, China. Email: yubaoguan@163.com; nanshan@vip.163.com.

Background: The differentiation of benign and malignant solitary pulmonary nodules (SPNs), especially subsolid nodules, is still challenging because of the small size, slow growth, and atypical imaging characteristics of these nodules. We aimed to determine the significance of mass growth rate (MGR) and mass doubling time (MDT) at follow-up CT of malignant SPNs.

Methods: This retrospective study included 167 patients (169 SPNs, diameter 8-30 mm). Among the 169 SPNs, 114 malignant SPNs were classified into three types: pure ground-glass nodules (pGGNs), part-solid nodules (pSNs), and solid nodules (SNs). These patients were followed up for at least 3 months. Threedimensional manual segmentation was performed for all these nodules, and the intra- and inter-observer variabilities of diameter, volume, and mass measurement were assessed. From initial and follow-up CT scans, growth rates of the diameter, volume, and mass of the SPNs were compared. MDT and volume doubling time (VDT) were calculated and were compared among groups.

Results: Mass measurements had the best inter-observer consistency and intra-observer repeatability; the coefficients of variation of the mass measurements were the smallest. The mean growth rates of the diameter, volume, and mass of pGGNs, pSNs, and SNs significantly differed at different time points $(\mathrm{P}<0.001)$. Mean MDTs and VDTs of pGGNs, pSNs, and SNs were 655 vs. 848 days, 462 vs. 598 days, and 230 vs. 267 days, respectively $(\mathrm{P}<0.05)$.

Conclusions: Mass measurements are an objective and accurate indicator in SPN assessment. During a 2-year follow-up, the mean growth rates of the diameter, volume, and mass of pGGNs, pSNs, and SNs differed at different time points, the greatest difference was observed in mean MGR. Mean MDT of malignant SPNs is less than the mean VDT.

Keywords: Solitary pulmonary nodule (SPN); lung cancer; mass doubling time (MDT); mass growth rate (MGR); volume doubling time (VDT)

Submitted Jan 04, 2018. Accepted for publication Mar 30, 2018.

doi: $10.21037 /$ jtd.2018.04.25

View this article at: http://dx.doi.org/10.21037/jtd.2018.04.25 


\section{Introduction}

Solitary pulmonary nodules (SPNs) are round or oval lesions that occur in the lungs, have a diameter $\leq 30 \mathrm{~mm}$, and are not accompanied by atelectasis, satellite lesions, or enlarged lymph nodes (1). Pulmonary nodules can be divided into solid and subsolid nodules, according to whether the lung parenchyma is completely covered by the nodule on CT imaging; subsolid nodules can be subdivided into pure ground-glass nodules (pGGNs) and part-solid nodules (pSNs) (2). Currently, the differentiation of benign and malignant SPNs is still challenging due to the small size, slow growth, and atypical imaging characteristics of these nodules. Specifically, its accuracy in the diagnosis of pulmonary nodules with a diameter of $>2$ and $\leq 2 \mathrm{~cm}$ has been reported as $82 \%$ and $61 \%$, respectively (3). Regular follow-up with CT scans may be used to identify visually detectable growth in these lesions, and to discriminate between benign and malignant pulmonary nodules.

In clinical practice, a general method to determine SPN size is two-dimensional measurement of the maximum nodule diameter. In this simple and easy to perform method, the maximum length of the nodule in a CT section is measured. However, computer-aided diagnostic software has greatly facilitated imaging tasks, and it has been proved that three-dimensional volume measurements are more accurate than two-dimensional measurements $(4,5)$. Furthermore, three-dimensional measurements can reveal increases in volume and therefore nodule growth and doubling time, both of which are important indicators of the differentiation of benign and malignant SPNs.

The growth of a pulmonary nodule involves changes in volume as well as in density. We have observed that in some lesions, such as subsolid nodules, the solid component gradually increases during follow-up, or solid nodules (SNs) may exhibit necrosis, liquefaction, or cavitation with little or no change in their diameter and volume. In these situations, diameter and volume measurements are insufficient to evaluate changes in the lesion. Mass measurements have been used to evaluate pulmonary alveolar proteinosis and recently used to assess SPNs $(6,7)$. Kim et al. (8) reported that mass measurements were also useful in the follow-up evaluation of partsolid pulmonary nodules (solid component $\leq 5 \mathrm{~mm}$ ). In addition, de Hoop et al. (9) found that mass measurements could reveal nodule growth earlier and more accurately than diameter and volume measurements. Song et al. (10) reported that pGGNs and part-solid GGNs with solid components of $\leq 5 \mathrm{~mm}$ showed significantly longer volume doubling time (VDTs) and mass doubling time (MDTs) than part-solid GGNs with solid components $>5 \mathrm{~mm}$. Thus far, the usefulness of mass growth rate (MGR) and MDT in the long-term follow-up of malignant subsolid and solid SPNs have rarely been reported. The purpose of our study was to determine the significance of MGR and MDT during the CT follow-up of malignant SPNs.

\section{Methods}

\section{Subjects}

The local institutional review board approved this retrospective study, and a waiver of informed consent was given. The patients we chose were all included in a medical image database of pulmonary nodules in our hospital. This study involved 167 patients with 169 CT-confirmed SPNs, who were treated between January 2011 and December 2016. All 169 SPNs were detected by chest CT (two patients had two SPNs each).

Of the 169 SPNs, 114 SPNs confirmed to be malignant nodules were included in the follow-up study. The exclusion criteria were as follows: patients with benign nodules or metastases, patients with nodules that showed shrank during follow-up, patients with incomplete information, patients who did not undergo surgery, and postoperative followup time $<3$ months. Follow-up CT was performed (a total of 177 CT scans over 2-year follow-up) at different times (3, 6, 12, 18, and 24 months). Patients were divided into three types according to the type of nodule: pGGNs $(n=29)$, pSNs $(n=45)$ and SNs $(n=40)$. Information about the 114 malignant SPNs is shown in Figure 1 and Table 1.

\section{CT scanning apparatus and parameters}

All 167 patients underwent chest CT. The Aquilion 16-slice CT machine (Toshiba, Tokyo, Japan) was used, and the scanning parameters were as follows: tube voltage, $120 \mathrm{kV}$; automatic tube current modulation; tube speed, $0.5 \mathrm{r} / \mathrm{s}$; pitch, 0.938; acquisition matrix, 512×512; and reconstruction section thickness, $2.0 \mathrm{~mm}$. During $\mathrm{CT}$, the patient was in a supine position. Scanning was performed from the bases to the apices of the lungs and was completed within a single maximal inspiratory breath-hold. A standard algorithm (FC51) was used for reconstruction, and measurements were conducted in the lung-tissue window. 


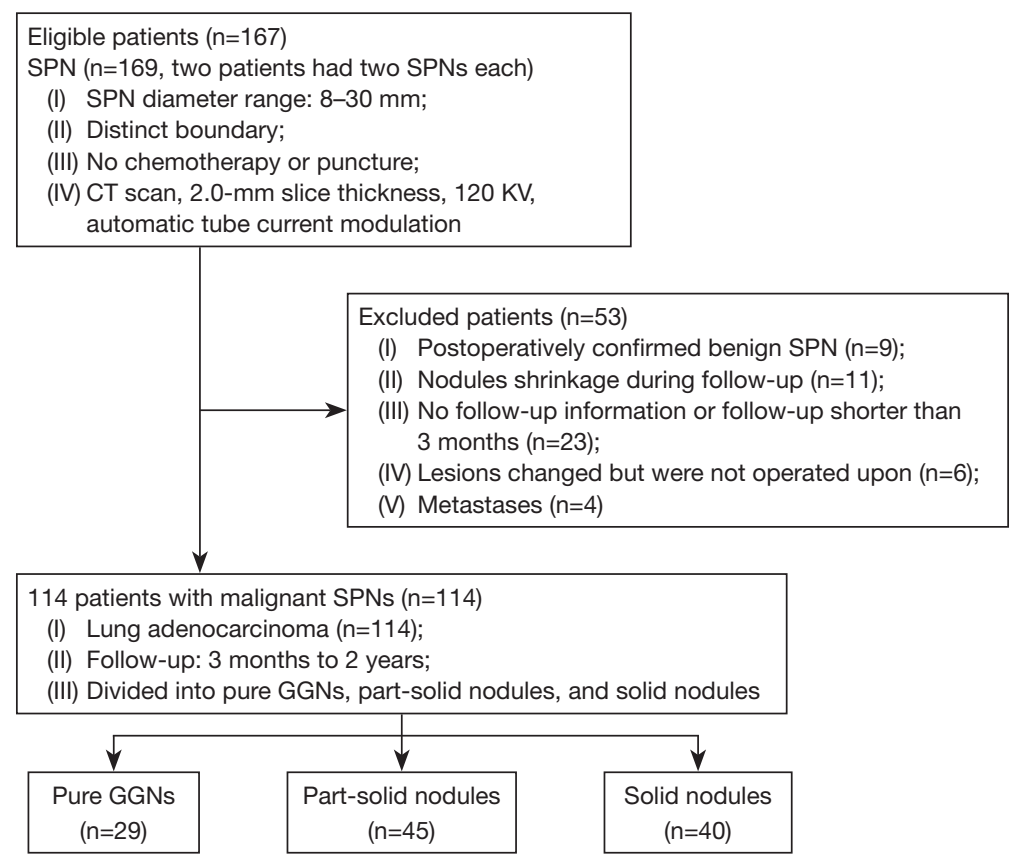

Figure 1 Enrollment and exclusion of subjects. SPN, solitary pulmonary nodule; GGN, ground-glass nodule

Table 1 Total number of CT scans of 114 malignant SPNs during 2 years of follow-up

\begin{tabular}{|c|c|c|c|c|}
\hline Time point (months) & \multicolumn{3}{|c|}{ Number of CT scans } & Total \\
\hline 3 & 13 & 16 & 12 & 41 \\
\hline 6 & 12 & 13 & 10 & 35 \\
\hline 12 & 11 & 14 & 11 & 36 \\
\hline 24 & 12 & 10 & 10 & 32 \\
\hline Total & 58 & 64 & 55 & $177^{\star}$ \\
\hline
\end{tabular}

*, total number of CT scans during 2 years of follow-up. SPN, solitary pulmonary nodule; GGN, ground-glass nodule.

\section{Image analysis and measurements of diameter, volume, and mass}

Two independent observers with $>5$ years of experience performed all measurements. Observer 1 repeated all measurements after an interval of 2 months to estimate intra-observer variability. Each observer independently categorized each SPN in the data set as pGGN, pSN, or $\mathrm{SN}$. The diameter of the SPN was determined by using the electronic calipers function of our picture archiving and communication system (Neusoft Medical Systems,
Shenyang, China) on the axial image in which the SPN had the greatest dimensions. Software for the quantitative analysis of pulmonary nodules (Emphylx, Department of Radiology/iCAPTURE Laboratory, University of British Columbia, Vancouver, BC, Canada) was used to manually outline the boundaries of the nodules in images in the DICOM format, with a section thickness of $2 \mathrm{~mm}$. After the successful outline and segmentation of all 114 SPNs, the volume and mass of the nodules were automatically calculated. 


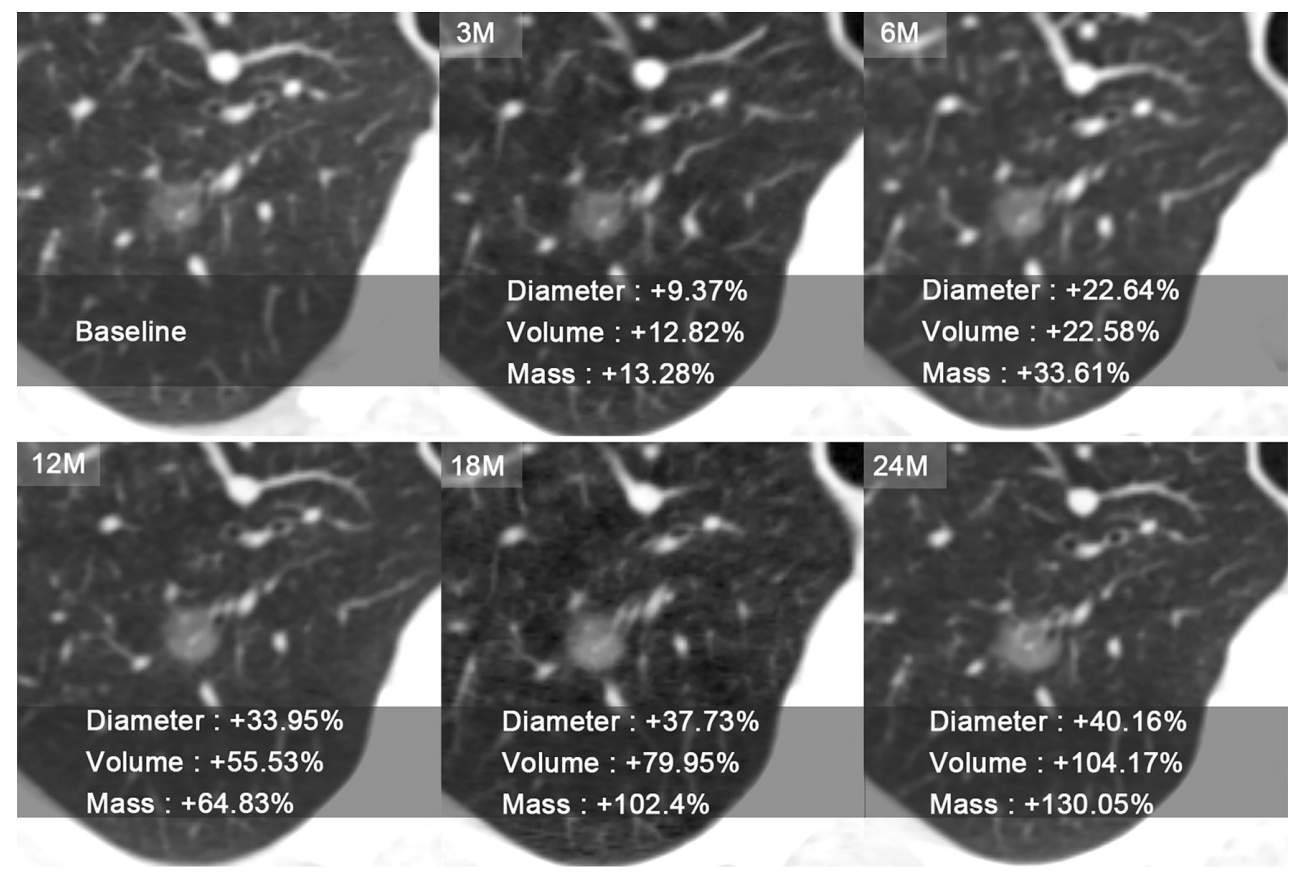

Figure 2 A-66-year-old man with a history of SPN in the right upper lobe. Diameter, volume, and mass of nodule were measured and analyzed at the baseline and during follow-up at 3, 6, 12, 18, and 24 months. Section thickness $=2 \mathrm{~mm}$.

\section{Predicting the growth rates of the diameter, volume, mass and the VDT and MDT of malignant SPNs}

For each follow-up nodule: first, we calculated the diameter growth rate (DGR), volume growth rate (VGR), and MGR. $\mathrm{DGR}=(\mathrm{D} 2-\mathrm{D} 1) / \mathrm{D} 1 \times 100 \% ; \mathrm{VGR}=(\mathrm{V} 2-\mathrm{V} 1) / \mathrm{V} 1 \times 100 \%$; MGR $=(M 2-M 1) / M 1 \times 100 \%$. D2 (or V2, M2) and D1 (or $\mathrm{V} 1, \mathrm{M} 1$ ) are the final and initial diameter (or volume, mass), respectively.

Second, VDTs and MDTs were calculated for volumeand mass-growing nodules by using an equation based on a modified Schwartz formula (10-12) of an exponential growth model, thus: $\mathrm{DT}=[\ln 2 \times \Delta \mathrm{T}] /[\ln (\mathrm{X} 2 / \mathrm{X} 1)]$, where DT is doubling time, $\mathrm{X} 2$ and $\mathrm{X} 1$ are the final and initial volumes (or mass), respectively, and $\Delta \mathrm{T}$ (days) is the interval between the two CT scans (10).

\section{Statistical analysis}

First, to determine variability of the diameter, volume, and mass measurements, we calculated the $95 \%$ confidence interval (CI) for the limits of agreement by using Bland-Altman analysis. To assess intra-observer variability, we compared the two measurements of observer 1; for inter-observer variability, the first measurement of observer 1 was compared with the measurement of observer 2. Second, to compare the degree of variability in the diameter, volume, and mass measurements, we calculated inter-observer and intraobserver coefficients of variation (CVs) of these three measurements. $\mathrm{CV}$ was calculated as the standard deviation divided by the mean. To compare intra-observer and interobserver CV for each method, a paired $t$-test was used.

For the 114 malignant SPNs, the DGR, VGR, and MGR of the nodules were calculated (Figure 2). A variance analysis model was used to study the effect of the measurement methods, nodule types, and follow-up time on nodular growth rates. We used logarithmic transformation of the growth rates to obtain normal distribution and used the variance analysis model to analyze and the Bonferroni method to test all pairwise comparisons among groups.

Two-related samples nonparametric tests were used to evaluate the difference of predicted VDTs and MDTs for each nodule type over time. The difference of predicted VDTs and MDTs of the three nodule types were compared by using the Kruskal-Wallis test, followed by Bonferroni correction for pairwise comparisons among the three types.

SPSS 20.0 software was used for statistical analyses. A P 
value $<0.05$ (two-tailed) indicated statistical significance.

\section{Results}

\section{Clinical and pathological manifestations}

This study involved 167 patients (91 men and 76 women), with a mean age of $61.2 \pm 9.5$ years (range, $41.0-89.0$ years). Of the 169 SPNs, nodule diameter ranged from 8 to $30 \mathrm{~mm}$, with a mean $( \pm S D)$ diameter of $15.79 \pm 8.47 \mathrm{~mm}$. One hundred and fourteen SPNs were confirmed to be malignant nodules - they were all adenocarcinomas.

\section{Variability of diameter, volume, and mass measurements}

The inter-observer and intra-observer Bland-Altman plots of the diameter, volume, and mass measurements of the 169 SPNs are shown in Figure 3. For the diameter measurements, the $95 \%$ CI of the inter-observer and intra-observer variability were $-1.3,1.2$ and $-0.95,0.79$, respectively. Both the volume and mass measurements were subjected to logarithmic transformation, and the $95 \%$ CIs of the inter-observer and intra-observer variability in the volume measurements were $-1.05,1.19$ and $-0.48,0.55$, respectively, while the corresponding values for the mass measurements were $-0.59,0.63$ and $-0.25,0.28$.

\section{Comparison of the CV of diameter, volume, and mass measurements}

The inter-observer and intra-observer CVs of the diameter, volume, and mass measurements of the 169 SPNs were 0.11 and $0.10,0.10$ and $0.07,0.07$ and 0.05 , respectively. There were significant differences in inter-observer and intra-observer CVs of the diameter, volume, and mass measurements $(\mathrm{P}<0.001)$, with the $\mathrm{CV}$ of the mass measurements being the smallest.

\section{DGR, VGR and MGR at different time points}

Mean DGR, VGR, and MGR of the 114 malignant SPNs at different time points during follow-up are shown in Table 2. Mean DGR, VGR, and MGR of the malignant SPNs showed little variation during the first 12 months of followup. However, VGR and MGR significantly increased at 18 and 24 months of follow-up (Figure 4); the most significant difference was in the MGR $(\mathrm{P}<0.05)$. Furthermore, all three types of growth rate were highest in SNs.
The results of the variance analysis model show that the $\mathrm{P}$ values of measurement methods, nodule types, and time points of follow-up were all less than 0.05 , and there was an interaction effect between nodule types and time points of follow-up (Table 3). By using the Bonferroni method to test all pairwise comparisons among groups, different measurement methods showed significant differences of diameter, volume, and mass (Table 4).

\section{VDTs and MDTs of malignant SPNs}

VDTs and MDTs of the 114 malignant SPNs were calculated. VDTs and MDTs of pGGNs ( $\mathrm{n}=29)$, pSNs $(\mathrm{n}=45)$, and $\mathrm{SNs}(\mathrm{n}=40)$ were mean $( \pm \mathrm{SD}) 848 \pm 330$ vs. $655 \pm 225$ days, $598 \pm 229$ vs. $462 \pm 183$ days, and $267 \pm 91$ vs. $230 \pm 73$ days, respectively. There was significant difference in predicted VDTs and MDTs over time in each type of SPN $(\mathrm{P}<0.05)$. A significant difference was found in predicted VDTs and MDTs of the three different types of SPNs ( $\mathrm{P}<0.001$, Table 5).

\section{Discussion}

In this study, the inter-observer consistency and intraobserver repeatability of diameter, volume, and mass measurements were excellent, especially in the case of the mass measurements. The mean coefficient of variation (CV) of the mass measurements was lower than those of the diameter and volume measurements, and the difference among these three indicators was significant. These results are consistent with the results of Kim et al. (8) and de Hoop et al. (9). Therefore, compared with diameter and volume measurements of pulmonary nodules, mass measurements were a more objective and accurate indicator.

During 2 years of follow-up, growth of the diameter, volume, and mass of malignant SPNs is progressive and slow. Therefore, in the case of some indeterminate SPNs, regular follow-up with CT scans (to observe size and density changes in the nodules) is a treatment strategy, and the combination of multiple CT indicators can compensate for each other and reduce measurement errors.

Subsolid and solid SPNs present differing growth rates. In this study, SPNs were divided into pGGNs, pSNs, and SNs for comparative analysis. An appropriate follow-up referral time can not only reduce radiation doses but also enable the early differentiation of benign and malignant nodules. According to the ACCP guidelines for diagnosis and management of lung cancer (3rd edition) (3): in the individual with a solid, indeterminate nodule that measures $>8 \mathrm{~mm}$ in 

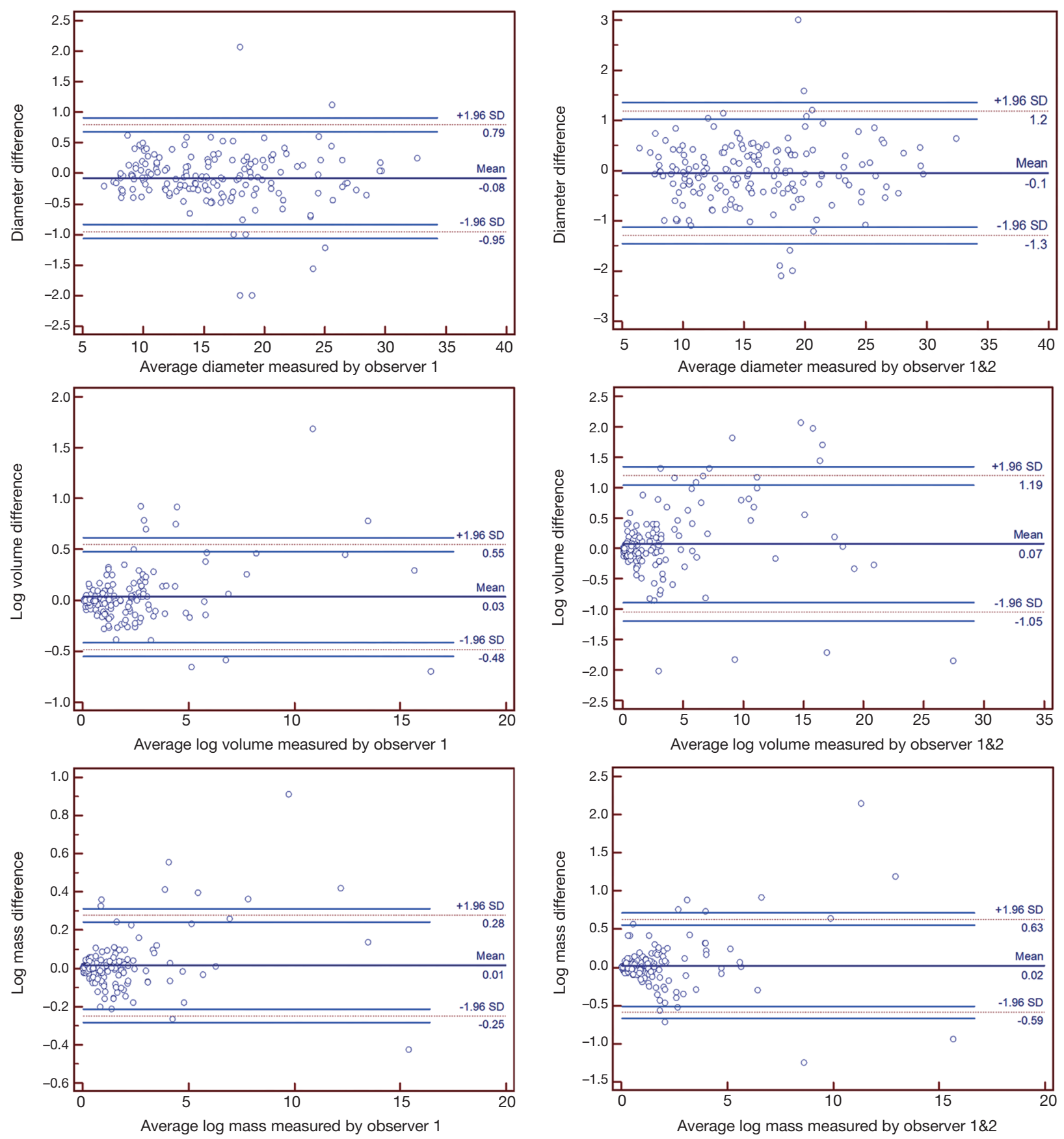

Figure 3 Inter-observer and intra-observer Bland-Altman plots of measurement variability in the 169 SPNs. SPN, solitary pulmonary nodule; SD, standard deviation. 
Table 2 Mean growth rates of the diameter, volume, and mass of the 114 malignant SPNs at different time points during follow-up (mean \pm SD)

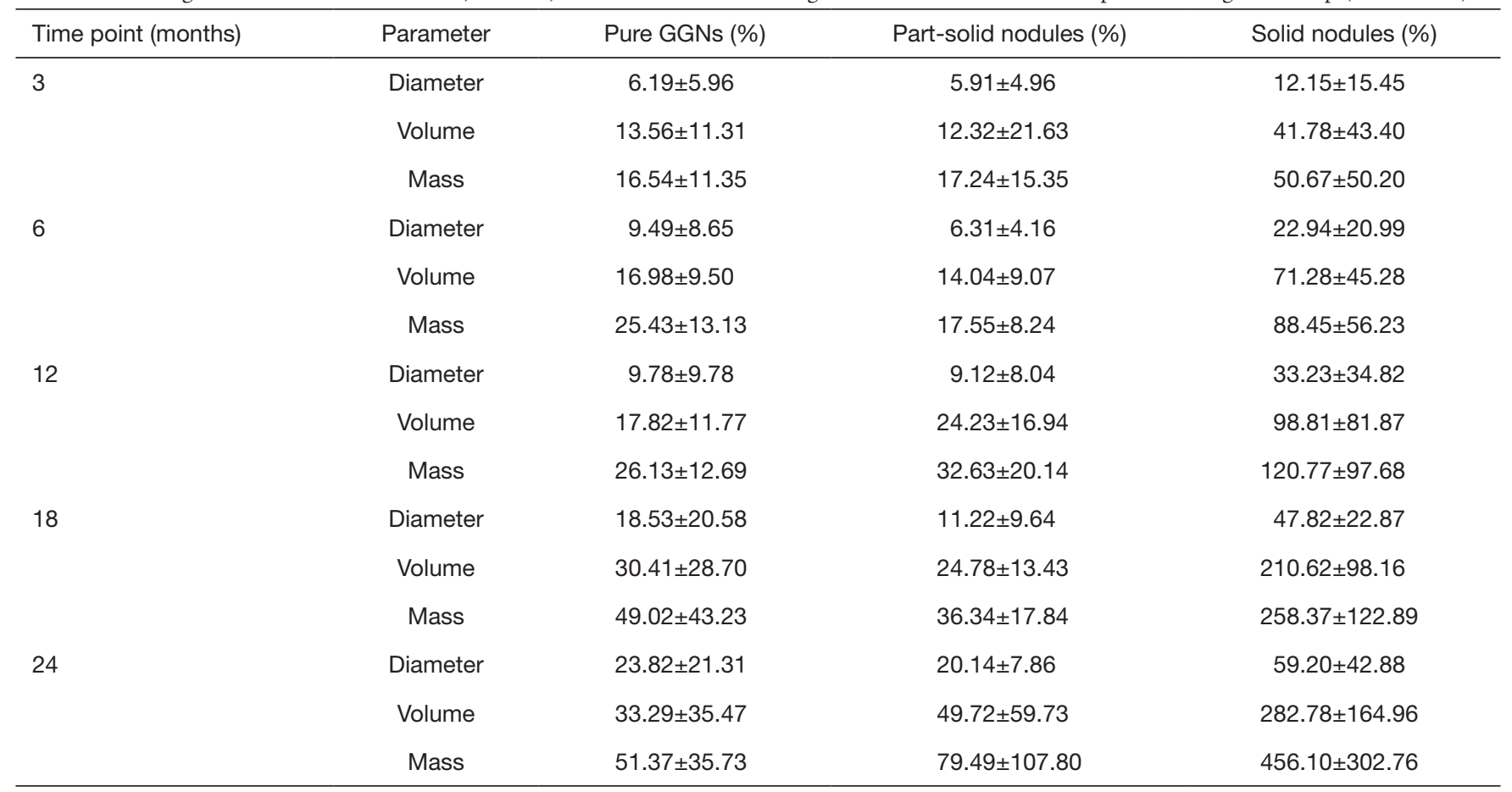

SPN, solitary pulmonary nodule; SD, standard deviation.
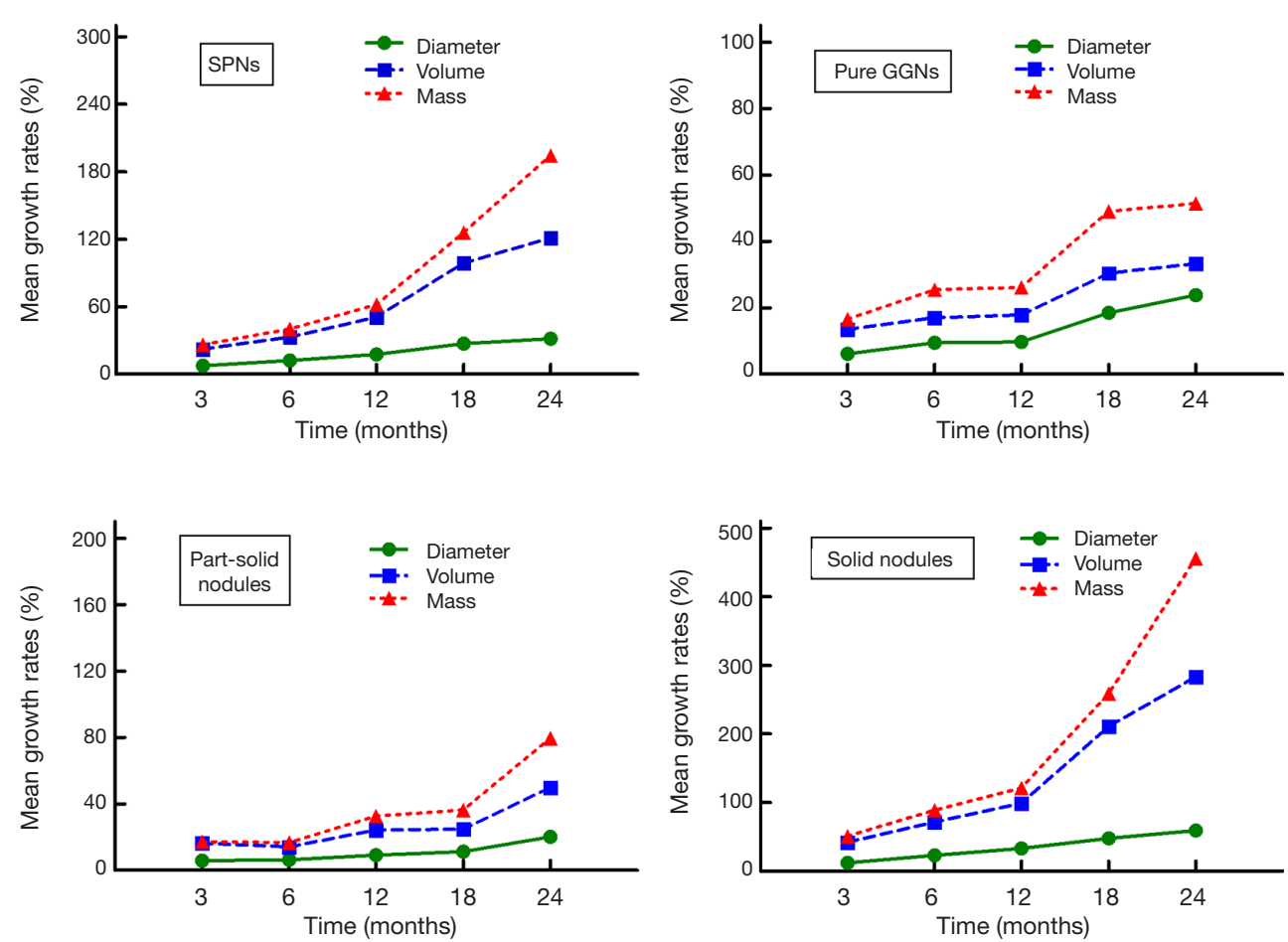

Figure 4 Mean growth rates of diameter, volume, and mass of different types of nodules with different follow-up intervals (\%). SPN, solitary pulmonary nodule; GGN, ground-glass nodule. 
Table 3 The results of the variance analysis model of measurement methods, types of nodule, and time points of follow-up

\begin{tabular}{lcccc}
\hline Source & Type III sum of squares & Mean square & F & 13.034 \\
\hline Corrected model & $97.679^{\mathrm{a}}$ & 2.220 & 4395.773 & 0.000 \\
Intercept & 748.706 & 748.706 & 118.459 & 0.000 \\
Type & 40.353 & 20.176 & 82.059 & 0.000 \\
Method & 27.953 & 13.977 & 31.219 & 0.000 \\
Time point & 21.270 & 5.317 & 0.348 & 0.018 \\
Type * time point & 3.200 & 0.400 & 2.348 \\
\hline
\end{tabular}

${ }^{\mathrm{a}}, \mathrm{R}^{2}=0.584$ (adjusted $\mathrm{R}^{2}=0.539$ ).

Table 4 Pairwise comparisons of different measurement methods

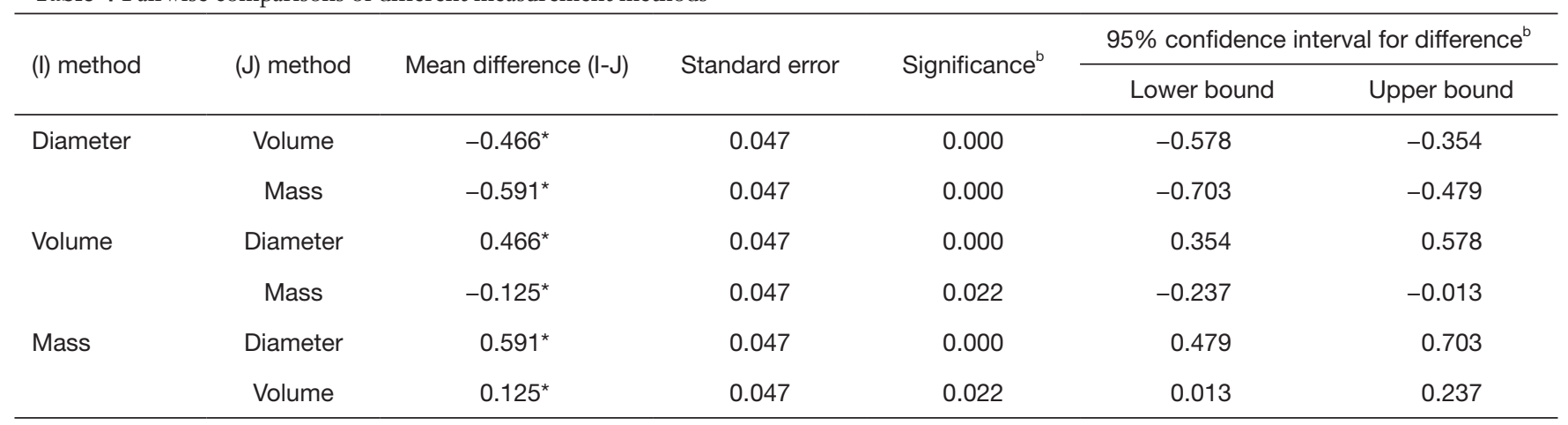

Based on estimated marginal means. ${ }^{*}$, the mean difference was significant at the 0.05 level; ${ }^{b}$, adjustment for multiple comparisons: Bonferroni.

Table 5 Doubling times in volume and mass of the different nodule types.

\begin{tabular}{lccc}
\hline Nodule types & VDT (mean \pm SD) (days) & MDT (mean \pm SD) (days) & P value \\
\hline Pure GGNs & $848 \pm 330$ & $655 \pm 225$ & 0.002 \\
Part-solid nodules & $598 \pm 229$ & $462 \pm 183$ & 0.001 \\
Solid nodules & $267 \pm 91$ & $230 \pm 73$ & $<0.001$ \\
P value & $<0.001^{* \# \$}$ & $<0.001^{* \# \$}$ & - \\
\hline
\end{tabular}

The data formats of VDT and MDT are mean \pm SD. * $\mathrm{P}<0.05$, doubling times of pure GGNs versus part-solid nodules; *, P<0.05, doubling times of pure GGNs versus solid nodules; $\$, P<0.05$, doubling times of part-solid nodules versus solid nodules. VDT, volume doubling time; MDT, mass doubling time; SD, standard deviation; GGN, ground-glass nodule.

diameter who undergoes surveillance, serial CT scans should be performed at 3 to 6,9 to 12 , and 18 to 24 months; in the individual with a non-SN measuring $>8-10 \mathrm{~mm}$ in diameter, repeat chest CT at 3 months should be performed. Therefore, we selected the time points $3,6,12,18$, and 24 months to observe subtle changes in the diameter, volume, and mass of SPNs. Mean growth rates of the diameter, volume, and mass differed significantly between pGGNs, pSNs, and SNs. The most significant difference was in MGR.

This study showed that the diameter, volume, and mass of SPNs varied very little during the first 12 months of follow-up, but both volume and mass significantly increased at 18 and 24 months. The growth rate of the diameter, volume, and mass of SNs was higher than those of subsolid nodules, which may be related to inert behavior and very slow growth in the early stage of the latter. However, the 
growth rate of subsolid nodules significantly increased in the later stages. The Fleischner Society Guidelines recommended that at least a 2-year follow-up was required to determine the malignancy of SNs, while a follow-up of at least 5 years is necessary for subsolid nodules (11).

Ravenel et al. (12) reported that for pulmonary nodules with a diameter $\leq 6 \mathrm{~mm}$, the reconstruction thickness should be $<1.25 \mathrm{~mm}$, but for pulmonary nodules with a diameter $\geq 8 \mathrm{~mm}$, a reconstruction thickness of $2.5 \mathrm{~mm}$ has little effect on the volume measurement. To more accurately reflect the variability of SPN diameter, volume, and mass measurements, we selected a reconstruction section thickness of $2 \mathrm{~mm}$. We conducted comparative analyses of the data obtained prior to and during follow-up (a longitudinal comparison) to reduce the impact of these factors on mass measurements.

Some reports have been published on VDTs of pulmonary nodules $(4,13-15)$. SNs whose volume remains stable for 2 years are generally considered to be benign. The doubling time of the most malignant $\mathrm{SNs}$ is between 30 and 400 days, while that of subsolid pulmonary nodules is longer than 400 days. In our study, mean VDTs were 848 days for pGGNs, 598 days for pSNs, and 267 days for SNs. Hasegawa et al. (16) reported that mean VDTs were 813 days for pGGNs, 457 days for pSNs, and 149 days for SNs. This difference may be due to the different number of each type of nodule in the two studies. In the earlier study (16), 61 lesions were divided into three types: pGGNs, 19 cases; pSNs, 19 cases; and SNs, 23 cases. In our study (114 total cases) there were 29 pGGNs, 45 pSNs, and 40 SNs, respectively. They followed-up for 3 successive years, while our study involved 2-year follow-up.

In our study, mean VDTs and MDTs of pGGNs, pSNs, and SNs were 848 vs. 655 days, 598 vs. 462 days, and 267 vs. 230 days, respectively. Thus, mean MDT was less than mean VDT. This result is consistent with the results of Song et al. (10). However, in their study, median MDT was 1,556.1 days for pGGNs, 1,199.9 days for solid components $\leq 5 \mathrm{~mm}$ and 627.7 days for part-solid GGNs with solid components $>5 \mathrm{~mm}$. The mean MDTs in our study were substantially lower than their results. This difference may be due to the different numbers, subgroups and followup time of the studies. Our study included only malignant SPNs and followed-up for 2 years, while their study included more indolent SSNs, and the follow-up time was longer. In addition, they found that a pairwise comparison between VDTs and MDTs did not reach significance, but a significant difference was found in predicted VDTs and
MDTs of the three different types of SPNs in our study.

Some limitations of this study exist. First, subsolid nodules were measured using a manual outline sectionby-section, which is time-consuming. Second, mass measurements are associated with volume and density. Therefore, we should further evaluate factors affecting the accuracy of CT density measurements. Third, there was a relatively small number of growing nodules for the calculation of VDT and MDT. Fourth, our study only included adenocarcinomas, other types of malignant SPN and benign SPNs should be addressed in future research. Fifth, the follow-up time should be extended to 5 years according to the 2017 Fleischner Society Guidelines (11). Last but not least, this was a retrospective study, and therefore several biases may apply.

In summary, comparison of quantitative CT indicators reveals that mass can be used as an objective and accurate indicator of SPN growth that has better consistency and repeatability than diameter and volume measurements. During a 2-year follow-up, MGR was significantly greater than diameter or volume growth rates in pGGNs, pSNs, and SNs. The diameter, volume, and mass of SPNs varied very little during the first 12 months of follow-up, but both volume and mass significantly increased at 18 and 24 months. Diameter, volume, and MGRs of SNs were higher than those of subsolid nodules. Mean MDTs of malignant SPNs were less than mean VDTs. Therefore, mass is a novel parameter for the evaluation of the growth rate of SPNs and can be used in the CT follow-up of pulmonary nodules.

\section{Acknowledgements}

Funding: The research was supported by Open Project of State Key Laboratory of Respiratory Disease (No. SKLRD2016OP011) and Science and Technology Planning Project of Guangdong Province (grant No. 2014A020212340 and 2017A040405065).

\section{Footnote}

Conflicts of Interest: The authors have no conflicts of interest to declare.

Ethical Statement: This retrospective study was approved by the Institutional Review Board of the First Affiliated Hospital of Guangzhou Medical University [Medical Ethical Review (MER) 2017-38], which waived the informed consent. 


\section{References}

1. Viggiano RW, Swensen SJ, Rosenow EC 3rd. Evaluation and management of solitary and multiple pulmonary nodules. Clin Chest Med 1992;13:83-95.

2. Henschke CI, Yankelevitz DF, Mirtcheva R, et al. CT screening for lung cancer: frequency and significance of part-solid and nonsolid nodules. AJR Am J Roentgenol 2002;178:1053-7.

3. Gould MK, Donington J, Lynch WR, et al. Evaluation of individuals with pulmonary nodules: when is it lung cancer? Diagnosis and management of lung cancer, 3rd ed: American College of Chest Physicians evidence-based clinical practice guidelines. Chest 2013;143:e93S-e120S.

4. Yankelevitz DF, Reeves AP, Kostis WJ, et al. Small pulmonary nodules: volumetrically determined growth rates based on CT evaluation. Radiology 2000;217:251-6.

5. Revel MP, Lefort C, Bissery A, et al. Pulmonary nodules: preliminary experience with three-dimensional evaluation. Radiology 2004;231:459-66.

6. Guan Y, Zeng Q, Yang H, et al. Pulmonary alveolar proteinosis: quantitative CT and pulmonary functional correlations. Eur J Radiol 2012;81:2430-5.

7. Perez A 4th, Coxson HO, Hogg JC, et al. Use of CT morphometry to detect changes in lung weight and gas volume. Chest 2005;128:2471-7.

8. Kim H, Park CM, Woo S, et al. Pure and part-solid pulmonary ground-glass nodules: measurement variability of volume and mass in nodules with a solid portion less than or equal to $5 \mathrm{~mm}$. Radiology 2013;269:585-93.

Cite this article as: Li J, Xia T, Yang X, Dong X, Liang J, Zhong N, Guan Y. Malignant solitary pulmonary nodules: assessment of mass growth rate and doubling time at follow-up CT. J Thorac Dis 2018;10(Suppl 7):S797-S806. doi: 10.21037/ jtd.2018.04.25
9. de Hoop B, Gietema H, van de Vorst S, et al. Pulmonary ground-glass nodules: increase in mass as an early indicator of growth. Radiology 2010;255:199-206.

10. Song YS, Park CM, Park SJ, et al. Volume and mass doubling times of persistent pulmonary subsolid nodules detected in patients without known malignancy. Radiology 2014;273:276-84.

11. MacMahon H, Naidich DP, Goo JM, et al. Guidelines for Management of Incidental Pulmonary Nodules Detected on CT Images: From the Fleischner Society 2017. Radiology 2017;284:228-43.

12. Ravenel JG, Leue WM, Nietert PJ, et al. Pulmonary nodule volume: effects of reconstruction parameters on automated measurements--a phantom study. Radiology 2008;247:400-8.

13. Aoki T, Nakata H, Watanabe H, et al. Evolution of peripheral lung adenocarcinomas: CT findings correlated with histology and tumor doubling time. AJR Am J Roentgenol 2000;174:763-8.

14. Kostis WJ, Yankelevitz DF, Reeves AP, et al. Small pulmonary nodules: reproducibility of three-dimensional volumetric measurement and estimation of time to followup CT. Radiology 2004;231:446-52.

15. Lindell RM, Hartman TE, Swensen SJ, et al. 5-year lung cancer screening experience: growth curves of 18 lung cancers compared to histologic type, CT attenuation, stage, survival, and size. Chest 2009;136:1586-95.

16. Hasegawa M, Sone S, Takashima S, et al. Growth rate of small lung cancers detected on mass CT screening. Br J Radiol 2000;73:1252-9. 